\title{
A randomized multicenter study comparing seawater washes and carmellose artificial tears eyedrops in the treatment of dry eye syndrome
}

This article was published in the following Dove Medical Press journal:

Clinical Ophthalmology

\author{
Manuel Diaz-Llopis ${ }^{1-5}$ \\ Maria Dolores \\ Pinazo-Duran ${ }^{2,3,6-9}$ \\ Loreto Diaz-Guiñon ${ }^{5}$ \\ Miriam Rahhal-Ortuño ${ }^{10}$ \\ Mercedes Perez-Ramos 8,9 \\ Rosabel Bosch ${ }^{8,9}$ \\ Roberto Gallego-Pinazo 11,12 \\ Rosa Dolz-Marco'1,12 \\ Teresa Diaz-Guiñon ${ }^{5}$ \\ María Diaz ${ }^{1,5}$ \\ Francisco Javier Romero ${ }^{13}$ \\ Angel Cisneros ${ }^{10}$ \\ 'University of Valencia, Valencia, \\ Spain; ${ }^{2}$ Ophthalmology Unit, \\ Faculty of Medicine, University of \\ Valencia, Valencia, Spain; ${ }^{3}$ Molecular \\ Ophthalmology Unit, Faculty of \\ Medicine, University of Valencia, \\ Valencia, Spain; ${ }^{4}$ Consellería de Sanitat \\ (Valencian Regional Health Ministry), \\ Valencian Autonomous Government, \\ Valencia, Spain; ${ }^{5}$ OVA - Valencia \\ Ophthalmology Institute, Valencia, \\ Spain; ${ }^{6}$ Hospital Universitario Doctor \\ Peset, Valencia, Spain; ${ }^{7}$ Santiago \\ Grisolía Eye Research Laboratory, \\ FISABIO, Valencia, Spain; ${ }^{8}$ Specialised \\ Medical Centre Alboraya, Valencia, \\ Spain; ' $S p e c i a l i s e d$ Medical Centre \\ Monteolivete, Valencia, Spain; \\ ${ }^{10}$ Department of Ophthalmology, La \\ Fe Hospital, Valencia, Spain; "IMED \\ Hospital, Valencia, Spain; ${ }^{2}$ Oftalvist \\ Clinic, Valencia, Spain; ${ }^{13}$ European \\ University of Valencia, Valencia, Spain
}

Correspondence: Manuel Diaz-Llopis University of Valencia, Avenida de Blasco Ibáñez, 13, 46010 Valencia, Spain

Tel +34 666530085

Email manuel.diaz@uv.es
Purpose: To investigate the safety and efficacy of sterile isotonic seawater washes vs standard treatment with carmellose artificial tears in dry eye syndrome (DES).

Patients and methods: This is a randomized multicenter prospective study with 12 weeks of follow-up. A group of patients with DES $(\mathrm{N}=60)$ were treated with seawater spray (Quinton $\left.{ }^{\circledR}\right)$ five times daily, and another similar group $(\mathrm{N}=60)$ were treated with carmellose artificial tears eyedrops (Viscofresh ${ }^{\circledR} 0.5 \%$ ) five times a day. The parameters studied and measured were as follows: Ocular Surface Disease Index questionnaire score, Schirmer I test (without anesthesia) score, tear osmolarity (TearLab ${ }^{\circledR}$ ), tear breakup time, tear meniscus height (meniscography OCT), fluorescein corneal staining score (National Eye Institute scale), lissamine green conjunctival staining score, and levels of IL-1 beta and IL-6 in tears (Luminex ${ }^{\circledR} 200$ ).

Results: In the group treated with seawater, symptoms decreased by $68 \%$, and the decrease was $26 \%$ statistically superior to the group treated with carmellose artificial tears eyedrops $(P<0.001)$. Levels of IL-1 beta and IL-6 in tears significantly decreased in the seawater group compared to the carmellose artificial tears group $(19 \% / 17 \%$ vs $52 \% / 51 \%)(P<0.001)$. There were no statistically significant differences in the other measured parameters. There were no cases of poor tolerance or side effects.

Conclusion: Administration of seawater is more effective than treatment with carmellose artificial tears in reducing symptoms and pro-inflammatory molecules (IL-1 beta and IL-6) in tears of patients with DES.

Keywords: dry eye syndrome, isotonic seawater, artificial tears, carmellose, IL-1 beta, IL-6, quality of life

\section{Introduction}

Dry eye syndrome (DES) is currently defined as a multifactorial disease of the eye surface characterized by the loss of homeostasis - instability and hyperosmolarity - of the tear film and secondary inflammation, with eye symptoms such as blurred vision, eye pain, and irritation and difficulty performing daily activities such as driving or using a computer in a sustained manner. Its high and increasing prevalence in the aged population (affecting $40 \%$ of people over 70 s), chronic nature, and capacity to affect quality of daily life make it an important disease. ${ }^{1,2}$

The usual treatment strategies such as lubrication and hydration of the eye surface with artificial tears of different compositions (hyaluronic acid, carmellose, etc.) and densities (gel, etc.), associated measures to directly or indirectly increase surface lubrication (tear plugs, nasal neurostimulation), as well as use of anti-inflammatory drugs (corticoids, cyclosporine, tacrolimus) make treating DES a frustrating experience, 
often only resulting in brief, temporary, and mild relief of discomfort. ${ }^{3-5}$

Through its alkaline $\mathrm{pH}$, high concentrations of bicarbonate, potassium, and magnesium, and low level of sodium, seawater has proved effective in relieving skin and nasal mucosal symptoms, dermatitis, and dry, atrophic, and atopic rhinitis through different mechanisms, such as surfacemoisturizing effect, anti-inflammatory effect, and washing of detritus and pro-inflammatory molecules by dragging and sweeping them away. ${ }^{6-8}$

The present study evaluated the efficacy and safety of this isotonic seawater in reducing the signs and symptoms of moderate-to-severe DES compared to standard treatment with carmellose artificial tears eyedrops.

\section{Patients and methods}

The study was conducted in accordance with the ethical principles set forth in the Declaration of Helsinki and the Good Clinical Practice Guidelines. The study protocol and informed consent were reviewed and approved by the institutional review board of the University of Valencia before study initiation. Written informed consent was obtained from each patient before the start of the study, and power analysis was performed to justify the number of patients enrolled in the study. The study processes, including recruitment of patients, collection of patients' data, and Ocular Surface Disease Index (OSDI) evaluation, was conducted at multiple clinical sites in Valencia (Spain). All the examinations were conducted by the same examiner (MD), under standardized conditions of room illumination and temperature (between $20^{\circ} \mathrm{C}$ and $25^{\circ} \mathrm{C}$ ). The measurements of parameters were carried out after a period of 2-3 hours from the last application of the treatment.

\section{Study population and inclusion criteria}

Eligible patients were those aged 55-75 years, who had been diagnosed with DES within the preceding 3 months, and had dry eye-related symptoms that were present for $>3$ months before the screening examination, or who had a minimum OSDI score of 23 with moderate or severe DES. Other inclusion criteria were as follows: 1) tear film breakup time (TBUT) of $\leq 5$ seconds, 2) fluorescein corneal staining (FCS) score of $\geq 4$ on National Eye Institute (NEI) scale, and 3) Schirmer I test score at 5 minutes of $\leq 10 \mathrm{~mm}$. These criteria needed to be met at both the screening and baseline examination.

Exclusion criteria included the following: 1) presence of anterior ocular disease (such as neurotrophic keratitis or keratoconus); 2) continued use of eye drops; 3) presence of a punctal plug or its removal within 1 month before the screening examination; 4) surgery on the ocular surface or intraocular surgery within 3 months before the screening period; 5) use of drugs or therapies that were prohibited from the screening examination to the end of study treatment (steroids, immunosuppressants, antihistamines, any prescription or over-the-counter ophthalmic drugs, contact lenses, and any other treatment agent affecting the dynamics of tear fluid, including its nasolacrimal drainage process), and 6) presence of systemic disease including allergy, Sjögren's syndrome, or ocular graft-vs-host disease.

\section{Study design and randomization}

This is a prospective, randomized, multicenter, activecontrolled trial conducted in three phases: screening, evaluation, and follow-up. As much as possible, the investigators of the study were blinded to the treatment; however, complete blinding could not be accomplished because of the big difference between the packaging features of the different treatment agents. Patients were divided and assigned to two groups based on simple randomization. Group I was treated with seawater spray (Quinton ${ }^{\circledR}$ Ocular sterile isotonic seawater; Quinton Laboratories, Alicante, Spain) five times daily for 12 weeks (Figure 1). The sterile isotonic seawater was applied in the form of a spray a few centimeters from the patient's eye. The said isotonic solution was specially designed for application to the ocular surface. Group II was treated with carmellose artificial tears eyedrops (Viscofresh ${ }^{\circledR} 0.5 \%$; Allergan Inc., Irvine, CA, USA) five times daily for 12 weeks.

\section{Assessment of outcome measures Efficacy assessments}

The parameters studied and measured were as follows: OSDI questionnaire score, Schirmer I test (without anesthesia) score, tear osmolarity $\left(\right.$ TearLab $\left.^{\circledR}\right)$, TBUT, tear meniscus height (TMH) (meniscography optical coherence tomography [OCT]), FCS score (NEI scale), lissamine green conjunctival staining (LGCS) score (NEI scale), and levels of IL-1 beta and IL-6 in tears (Luminex ${ }^{\circledR} 200$ ).

The sum of the scores was used in the analysis. All of these parameters were assessed at baseline and week 12 .

All participants were asked to fill the OSDI questionnaire ${ }^{9-14}$ (Allergan Inc.) to distinguish those with normal ocular surface, and those with mild, moderate, or severe DES. Only those with moderate or severe DES were included in the present study. The overall OSDI score delineated the ocular surface as normal (0-12 points), with mild DES (13-22 points), 


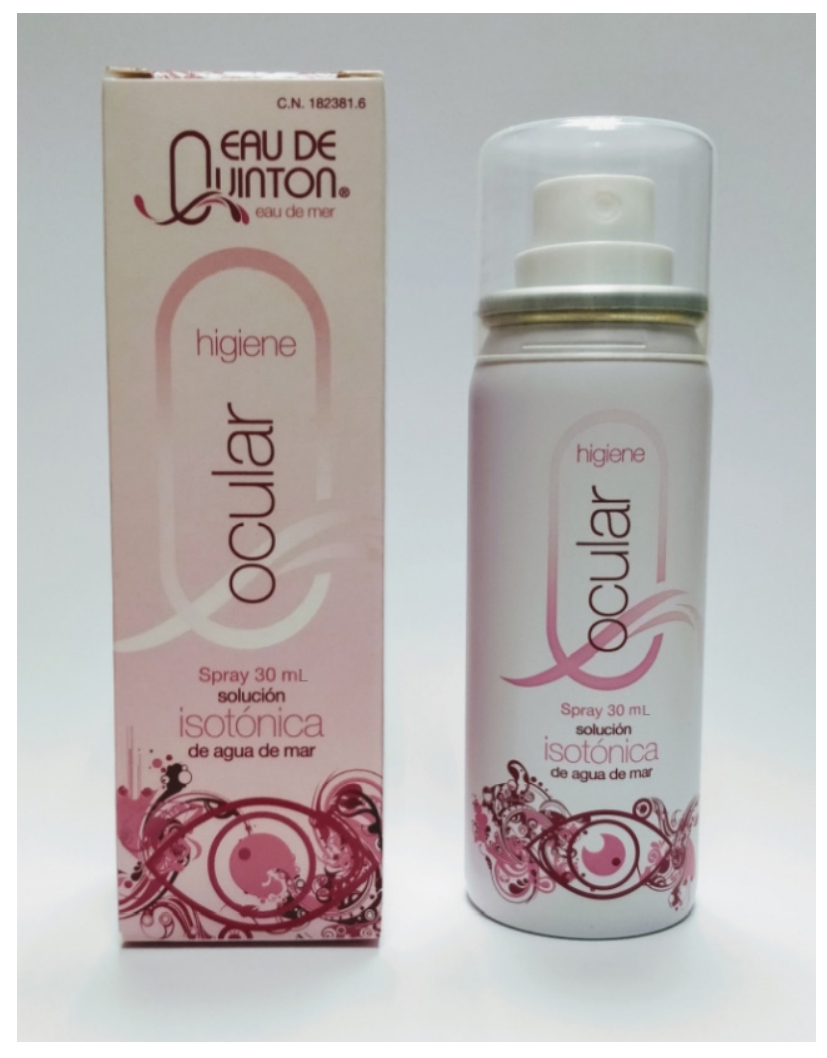

Figure I Quinton ${ }^{\circledR}$ Ocular isotonic seawater spray.

with moderate DES (23-32 points), and with severe DES (33-100 points). The OSDI questionnaire was filled out during the medical appointments.

For measuring tear osmolarity, the TearLab ${ }^{\circledR}$ osmolarity system (Equipsa, Doral, FL, USA) was used. The terminal was prepared to capture $50 \mu \mathrm{L}$ of tears and was applied without anesthesia to the outer and lower edge of the eye. Pathological osmolarity values were regarded as those $>316 \mathrm{mOsm} / \mathrm{L}\left(\right.$ TearLab $\left.^{\circledR}\right) .{ }^{15}$

For measuring TBUT, $5 \mu \mathrm{L}$ of $2 \%$ fluorescein solution was first instilled in the conjunctival sac. TBUT was then evaluated by slit-lamp microscopy. The elapsed time from a normal blink to the first appearance of a dry spot in the tear film was measured thrice. ${ }^{16}$

Schirmer I test was performed without anesthesia to measure tear volume as follows. A Schirmer test strip was placed on the lower eyelid between palpebral conjunctiva and bulbar conjunctiva without touching the cornea. The tear volume was then measured for 5 minutes. The length (in millimeters) of tear fluid absorbed on the strip measured from the edge of the strip was recorded as tear volume. ${ }^{17}$

$\mathrm{TMH}$, which is a sensitive indicator of the tear film volume and considered an important marker in the diagnosis of aqueous-deficient dry eye, was measured by meniscography OCT.
An RTVue-100 FD-OCT system (Optovue, Fremont, CA, USA) with a corneal adaptor module was used. This system has a $6 \mathrm{~mm}$ vertical beam that takes 26,000 axial scans per second and has a $5 \mathrm{~mm}$ axial resolution to a depth of $2.8 \mathrm{~mm}$. Vertical images were recorded three times for 3 seconds after each blink at the 6 o'clock position of the cornea. A built-in caliper was used to measure the TMH. The TMH was determined as the length from the point where the meniscus intersected with the cornea superiorly to the eyelid inferiorly. ${ }^{18}$

For calculating FCS scores, $20 \mu \mathrm{L}$ of $2 \%$ fluorescein solution was instilled in the conjunctival sac as the patient blinked normally. Corneal staining was examined under standard illumination using a slit-lamp microscope with a cobalt blue filter. According to the NEI/Industry Workshop report, the cornea was divided into five fractions. FCS scores were measured on a 0 - to 3-point scale, in the superior, inferior, nasal, temporal, and central corneal zones: 0 (no staining), 1 (mild superficial stippling), 2 (moderate punctate staining, including superficial abrasion of the cornea), and 3 (severe abrasion or corneal erosion, deep corneal abrasion, or recurrent erosion). The total score was then calculated.

For calculating LGCS scores, $20 \mu \mathrm{L}$ of $1 \%$ lissamine green solution was instilled in the conjunctival sac, and the conjunctiva was divided into six fractions. Conjunctival staining was evaluated under low illumination by slit-lamp microscopy and was scored from 0 to 3 for each fraction, and then summed to calculate the total score.

The levels of IL- 1 beta and IL- 6 in tears were measured using Luminex ${ }^{\circledR} 200$ as follows. ${ }^{19,20}$ Tear samples were collected from all participants and analyzed with biochemical techniques. The gentle rubbing method was used to obtain reflex tears from the inferior meniscus of both the eyes of the participants using a micro Pasteur pipette. Tear samples collected from both the eyes were immediately deposited in micro Eppendorf tubes to be frozen and stored at $-80^{\circ} \mathrm{C}$ until assaying a specific set of inflammatory mediators. The human panel of cytokines that was assayed in this study was composed of the following ILs: IL-1 beta and IL-6. The analyses were performed using the Luminex ${ }^{\circledR} 200$ multiplex system (Luminex, Austin, TX, USA). Polystyrene beads coupled covalently to specific antibodies were prepared to react with an approximate amount of $20 \mu \mathrm{L}$ of each tear sample (which contains an unknown amount of these molecules), or with a standard solution (having a known amount of molecules), at room temperature for 1 hour. A series of washes (to remove unbound proteins) were performed. Biotinylated detection antibody specific for a different epitope on the cytokine was added to the beads and incubated at room temperature 
for 30 minutes. Streptavidin-phycoerythrin (which binds to the biotinylated detection antibodies) was used to detect the reaction mixture. Next, the flow-based Bio-Plex (Bio-Rad Laboratories, Hercules, CA, USA) suspension array system was used to identify and quantify each antigen-antibody reaction. The assayed set of inflammatory molecules was identified by bead color and fluorescence, with fluorescently labeled reporter molecules associated with each target protein. Unknown cytokine levels were calculated automatically by the Bio-Plex Manager software (Bio-Rad Laboratories) using a standard curve derived from a recombinant cytokine standard. Tear levels of the cytokines were corrected for the initial total protein concentration and finally expressed as mean $\pm \mathrm{SD}$ of three independent measurements.

\section{Safety assessment}

The safety variable was the occurrence of adverse events, determined at various visits by means of physical signs and symptoms, external eye examination, slit-lamp microscopy, measurement of visual acuity and intraocular pressure, and funduscopy.

\section{Statistical analyses}

Statistical analysis was performed using SPSS version 18.0 (SPSS Inc., Chicago, IL, USA). The Kolmogorov-Smirnov test was performed to assess normality for continuous variables. The outcomes were compared between the two groups using an independent $t$-test for continuous variables or the chi-squared test for categorical variables. The comparisons of outcome measures between the baseline and 12 weeks after intervention in each group were performed using a paired $t$-test, and the differences in the degree of change were compared between the two groups using an independent $t$-test. To compare changes in Groups I and II, repeated measures ANOVA was used. A value of $P<0.05$ was considered significant.

\section{Results \\ Demographic data of the population studied}

A total of 193 participants were recruited into the trial. Sixty patients in Group I (seawater) and 60 patients in Group II (artificial tears) completed the entire protocol. The difference between the two groups in sex distribution was not significant $(P=0.447)$. The mean age of the participants in Group I and Group II was 68.08 \pm 6.29 and $66.83 \pm 8.42$ years, respectively, and there was no significant difference between the groups $(P=0.379)$ (Table 1).

\section{OSDI questionnaire}

The OSDI scores after the intervention showed a significant decrease compared to those before the intervention in both groups $(P<0.001)$. In addition, there was a significant difference in the efficacy of the two products $(P<0.001)$, with seawater spray wash being much more effective. The mean score improvement was $68 \%$ in the seawater spray group compared to $42 \%$ in the carmellose artificial tears eyedrops group; in other words, the mean score improvement was 26\% higher than in the artificial tears group (Table 2).

\section{Corneal and conjunctival staining}

The corneal and conjunctival staining scores after the intervention also showed a significant decrease compared to those before the intervention $(P<0.001)$. However, although the improvement of the seawater group was superior, the two products - seawater and artificial tears - did not show statistically significant differences (FCS score: $P=0.793$; LGCS score: $P=0.822$; Table 2).

The rest of the parameters studied were Schirmer I test (without anesthesia) score, tear osmolarity (TearLab ${ }^{\circledR}$ ), TBUT, and TMH (meniscography OCT) (Table 2).

Table I Demographic data

\begin{tabular}{l|l|l|l}
\hline & Group I (seawater) & Group II (artificial tears eyedrops) & P-value \\
\hline Age, mean \pm SD & $68.08 \pm 6.29$ & $66.83 \pm 8.42$ & 0.379 \\
Female:male & I.60:I & I.56:I & 0.447 \\
Severity, $\mathrm{n}$ & 37 & 39 & 0.829 \\
$\quad$ Moderate & 23 & 21 & \\
$\quad$ Severe & 14 & 12 & 0.238 \\
Hypertension, $\mathrm{n}$ & 19 & 17 & 0.658 \\
Cholesterol $>260 \mathrm{mg} / \mathrm{dL}, \mathrm{n}$ & 13 & 18 & 0.051 \\
Diabetes, $\mathrm{n}$ & 13 & 15 & 0.324 \\
Osteoarthritis, $\mathrm{n}$ & 16 & 14 & 0.287 \\
Smoking history, $\mathrm{n}$ & 19 & 20 & 0.376 \\
Cardiovascular disease, $\mathrm{n}$ & & & \\
\hline
\end{tabular}


Table 2 Comparison of the clinical efficacy of seawater and artificial tears eyedrops

\begin{tabular}{|c|c|c|c|c|c|c|c|c|}
\hline \multirow[t]{2}{*}{ Type of test } & \multicolumn{3}{|c|}{ Group I (seawater) } & \multicolumn{5}{|c|}{ Group II (artificial tears eyedrops) } \\
\hline & Baseline & I 2-week visit & $P$-value (I) & Baseline & I 2-week visit & $P$-value (I) & $P$-value (2) & $P$-value (3) \\
\hline OSDI score & $42.58 \pm 16.96$ & $23.94 \pm 15.19$ & $<0.001$ & $40.52 \pm 15.77$ & $28.5 \pm 16.19$ & $<0.001$ & 0.339 & $<0.001$ \\
\hline ConjScore & $7.67 \pm 2.98$ & $5.03 \pm 2.27$ & $<0.001$ & $7.43 \pm 2.20$ & $5.68 \pm 1.69$ & $<0.001$ & 0.334 & 0.822 \\
\hline CornScore & $1.23 \pm 1.43$ & $0.49 \pm 0.78$ & $<0.001$ & $1.15 \pm 1.18$ & $0.44 \pm 0.57$ & $<0.001$ & 0.807 & 0.793 \\
\hline TMH (mm) & $0.26 \pm 0.15$ & $0.28 \pm 0.13$ & 0.289 & $0.29 \pm 0.16$ & $0.27 \pm 0.09$ & 0.318 & 0.542 & 0.459 \\
\hline TO (mOsm/L) & $293.9 \pm 10.57$ & $296.4 \pm 9.50$ & 0.456 & $298.3 \pm 13.7$ & $295.6 \pm 7.2$ & 0.348 & 0.711 & $0.25 I$ \\
\hline TBUT (seconds) & $7 \pm 2.2$ & $7.64 \pm 2.60$ & 0.127 & $7.15 \pm 2.35$ & $8.09 \pm 2.74$ & 0.113 & 0.64 & 0.497 \\
\hline ST score $(\mathrm{mm})$ & $6.14 \pm 5.35$ & $6.36 \pm 5.66$ & 0.129 & $6.34 \pm 6.42$ & $7.10 \pm 6.20$ & 0.115 & 0.676 & $0.44 I$ \\
\hline
\end{tabular}

Notes: Group I: patients treated using seawater spray (Quinton ${ }^{\circledR}$ ); Group II: patients treated using carmellose artificial tears eyedrops (Viscofresh ${ }^{\circledR} 0.5 \%$ ). $P$-value (I) indicates the significance level of paired $t$-test within Group I or II between baseline and I 2 weeks after intervention; $P$-value (2) indicates the significance level of independent $t$-test for comparing between Groups I and II before intervention; $P$-value (3) indicates the significance level of independent $t$-test for comparing between Groups I and II after I 2 weeks. Abbreviations: ConjScore, conjunctival score; CornScore, corneal score; OSDI, Ocular Surface Disease Index; TBUT, tear breakup time; TMH, tear meniscus height; TO, tear osmolarity; ST, Schirmer test.

There were no significant differences before and after the administration of the two products in any of the other parameters tested.

\section{Multiplex analysis of inflammatory molecules in tears}

With the assayed amounts of tears utilized in the present study (mean $14 \pm 8 \mu \mathrm{L}$ ), it was possible to detect the majority of molecules related to inflammation (as in the human cytokine panel utilized herein) in $95 \%$ of the samples (Table 3). Polystyrene beads coupled covalently to specifically directed antibodies (cytokines) were allowed to react with each tear sample containing an unknown amount of the molecules, or with a standard solution containing a known amount of these molecules, at room temperature for 1 hour, following the manufacturer's instructions. Detection data of the inflammation molecules (expressed in $\mathrm{pg} / \mu \mathrm{L}$ ) from the tear samples are summarized in Table 3. When comparing Group I (seawater) vs Group II (carmellose eyedrops), the results showed statistically significant differences in the tear

Table 3 Levels of the inflammatory molecules IL-I beta and IL-6 in tears in Group I treated with isotonic seawater (Quinton ${ }^{\circledR}$ ) and in Group II treated with carmellose artificial tears eyedrops (Viscofresh $^{\circledR} 0.5 \%$ )

\begin{tabular}{|c|c|c|}
\hline & 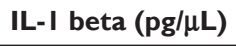 & IL-6 (pg/ $/ \mu \mathrm{L})$ \\
\hline \multicolumn{3}{|c|}{ Before starting/applying treatment } \\
\hline Seawater spray & $43.6 \pm 8$ & $32.5 \pm 6$ \\
\hline Carmellose artificial tears & $38.9 \pm 12$ & $36.4 \pm 8$ \\
\hline \multicolumn{3}{|l|}{ After treatment } \\
\hline Seawater spray & $8.3 \pm 3(19 \%)$ & $5.6 \pm 2(18 \%)^{* *}$ \\
\hline Carmellose artificial tears & $20.4 \pm 7(52 \%)$ & $18.6 \pm 6(51 \%)^{*}$ \\
\hline
\end{tabular}

Notes: Data are presented as mean \pm SD for all participants in each group, with decrease in levels shown in parentheses. Comparative analysis between groups: significance levels were taken at $P<0.01\left(^{*}\right)$ and $P<0.001$ (**). expression of IL-1 beta and IL-6. Levels of IL-1 beta and IL-6 in tears decreased significantly more in the seawater group compared to the carmellose artificial tears group (19\% IL-1 beta/17\% IL-6 vs 52\% IL-1 beta/51\% IL-6) $(P<0.001)$.

\section{Adverse events}

No cases of intolerance or side effects were observed in either of the two treatment groups.

\section{Discussion}

DES is the most frequent and prevalent ophthalmological disease worldwide and is likely to increase exponentially with the progressive aging of the population pyramid in the coming years and decades. Its degree of impact on quality of life is very high due to the continuity of discomfort caused throughout the day, year after year, and the consequent difficulties carrying out everyday activities (driving, reading, watching television, using computers, coping with air conditioning and heating in commercial and/or domestic interiors, street wind, and kitchens, etc.). The standard treatment is the frequent application of artificial tears to the eye surface. However, this only offers a very limited degree of effectiveness in relieving symptoms - especially the need for immediate relief understood as a "feeling of freshness" - and durability - a maximum of a few minutes - thus generating a permanent and generalized feeling of frustration in patients receiving this treatment. It merely prevents the disease from getting worse, without offering any sensation of improvement. Therefore, one of the main issues to be resolved is the need to find a truly gratifying treatment that generates a feeling of immediate and lasting relief in patients.

Another of the main issues to be solved is the chronification and the appearance of periodic inflammatory episodes secondary to the accumulation of inflammatory 
molecules - IL-1 beta, IL-6, etc. - on the conjunctival surface due to the lack of their neutralization, dilution, and entrainment (dragging), which generate an intense increase in the patient's discomfort, accompanied by itching, redness, and photophobia, and are also responsible for corneal complications (eg, secondary keratitis). Permanent maintenance treatment with corticoids, immunosuppressants, or autologous serum solutions in the form of eye drops is complex and can cause side effects (eg, bacterial superinfections). Given the necessary high frequency of application and low degree of efficacy of the current treatment options, as well as the potential side effects of anti-keratitis treatments, the introduction of new treatments is necessary. Ideally, new treatments should be easily and frequently attainable, and easy for the patients to get hold of and self-administer, as would be the case with seawater.

Seawater has been used for years for the relief of mucosal and skin symptoms, especially dry, atrophic, psoriatic, and atopic dermatitis, chronic nonhealing trophic skin wounds and ulcers, and atrophic and allergic rhinitis, due to its documented therapeutic effects. There is also traditional nonscientific cultural information on its effectiveness in treating dryness and eye irritation - the classic technique of washing with water and salt, etc. Having reviewed the data on PubMed from the last 50 years, there is no documented scientific research on the efficacy of seawater in any ophthalmic disease in general, or in DES in particular. All the above data supported the initiation and performance of a scientific study to establish the probable efficacy of isotonic seawater in DES and the safety of its application and to establish its comparative efficacy vs standard treatment with carmellose artificial tears eyedrops.

In the group treated with seawater spray, the clinical symptoms of dry eye improved on average by $68 \%$, and the improvement was $26 \%$ more than in the group treated with carmellose artificial tears eyedrops, demonstrating the superiority of the seawater treatment. It should be noted that not all dry eye symptoms responded equally, with maximum efficacy shown on the gritty sensation, foreign body sensation, dryness, itching, burning, irritation, and redness, and with much less impact on the sensation of pain, need to blink and keep the eyes closed, or severe difficulty opening them in the morning. In addition, the perfect tolerance and complete absence of even minimal side effects supports the inclusion of seawater washing as the first basic therapeutic step in the initial treatment of dry eye. And this important fact is due to its special ability to achieve a sensation of freshness and immediate relief of symptoms, as well as the reduction of mucous secretions after its application as reported by the patients.

All the objective parameters related to changes or improvements in the production and quantity of tear film - osmolarity, TBUT, and the results of Schirmer I test, Schirmer II test, and OCT meniscography - did not change when applying either the seawater or artificial tears treatments, as there is no pathophysiological mechanism to influence their production. It is therefore clear that the great efficiency demonstrated by seawater spray has other fundamental mechanisms to explain its effectiveness.

The fact that there is a direct relationship in the decrease of pro-inflammatory molecules in the tears of patients with dry eye, and that there is a proven correlation between greater or lesser clinical efficacy in the improvement of symptoms with a greater or lesser decrease in the high pathological levels of these molecules, clearly indicates that this may be the main mechanism of action of seawater on DES. The documented 26\% (OSDI score) greater clinical efficacy of seawater compared to artificial tears statistically correlated with the greater decrease in IL-1 beta and IL-6 levels (by $19 \% / 17 \%$ with seawater compared to $52 \% / 51 \%$ with artificial tears).

Seawater has been shown to reduce the presence of IL-1 beta and IL- 6 by nearly half, and to levels virtually similar to those of the normal population without dry eye, and this lays the biochemical foundation for why it is so effective in the clinic, as well as the need for long-term studies that continuously demonstrate its ability to break the vicious circle of the pathogenesis of the disease, using its continuous and longterm administration to interrupt the episodes of exacerbation (keratitis, red eye, etc.) and end its chronicity. ${ }^{21,22}$ In addition, one should not forget that in these multiple mechanisms of seawater including neutralization, dilution, and entrainment, entrainment is unique and differentiates seawater from any other dry eye treatment and could explain much of its effectiveness. Lubricating, which is what artificial tears do, is not the same as washing by dragging (entrainment), which is what seawater does, just as it is not the same to wash your hands as it is to shower or to bathe in seawater. This would explain why the seawater spray has that special capacity to provide a sensation of freshness and immediate relief of symptoms, as well as reduce mucous secretions, which is not documented with any other treatment.

The fact that the microulcerations of the conjunctival and corneal surface (keratitis), documented by staining with sodium and lissamine green fluorescein stain, are reduced with isotonic seawater, and in a manner similar to that 
demonstrated by artificial tears, corroborates and confirms their healing and restoring capacity for the normalization of the ocular surface. The efficacy of seawater has already been documented in other dry and inflammatory diseases of other mucous membranes of the human body (dermatitis, trophic skin ulcers, rhinitis), but until the present study it had not been documented in any ophthalmological diseases, such as dry eye.

Among the therapeutic mechanisms of seawater that make it effective are: ${ }^{23-28} 1$ ) its alkaline $\mathrm{pH}$ and higher bicarbonate level, which, by decreasing the acidification of the inflammatory damaged surface, would promote tropism, healing, and restoration of mucosal integrity through increased levels of EGF; 2) its restoration of the electrolyte balance of the surface through its low sodium levels, eliminating or reducing the electrolyte imbalance that is at the origin of the pathophysiological chain of dry eye; 3 ) its anti-inflammatory effect due to its high concentration of potassium and magnesium, as it is able to reduce the levels of TNF and IL-8 already known, and from the present study, of IL-1 beta and IL-6, and thus end the vicious circle chronically perpetuating the dry eye; 4) its hydrating effect, preventing hyperosmolarity from the outset, provided that it is applied in a sufficiently frequent and maintained manner; and 5) its washing and cleaning effect by "dragging" and removing from the surface much of the cellular debris, hyperosmolarizing electrolytes, and pro-inflammatory molecules and cells (IL-1 beta, IL-6, IL-8, and MMP-3 and MMP-9, TNF, and macrophages and T-lymphocytes) through a mechanical sweeping mechanism, or "showering/bathing in seawater" effect.

\section{Limitations}

This study has some limitations. First, the duration was short. It may be insufficient for comparing the impact of the seawater and artificial tears treatments on long-term dry eye disease since seawater is believed to affect subconjunctival inflammation, which may take 3-6 months to cut the chronicity of the disease. However, while this study is limited in duration, its results are still evident and valid. A more extensive and long-term study is warranted to address important clinical issues more thoroughly.

\section{Conclusion}

This study showed that isotonic seawater solution was highly effective - clinically, histologically, and biochemically - and completely safe, with no side effects, in the treatment of DES. Its efficacy was achieved by reducing the symptomatology by $68 \%-26 \%$, which was more than the reduction achieved by artificial tears. The levels of IL-1 beta and IL-6 in tears decreased statistically superiorly in the seawater group compared to the carmellose artificial tears group (19\% IL-1 beta/17\% IL-6 vs 52\% IL-1 beta/51\% IL-6) $(P<0.001)$.

\section{Acknowledgment}

This study was supported by the Thematic Network for Cooperative Research in Health (RETICS), Carlos III Institute, Spanish Ministry of Health, Madrid, Spain.

\section{Disclosure}

The authors report no conflicts of interest in this work.

\section{References}

1. Am L, Lemp AM. The definition and classification of dry eye disease report of the definition and classification Subcommittee of the International dry eye workshop (2007). Ocul Surf. 2007;5(2):75-92.

2. Moss SE, Klein R, Klein BE. Prevalence of and risk factors for dry eye syndrome. Arch Ophthalmol. 2000;118(9):1264-1268.

3. Liu Y, Hirayama M, Cui X, Connell S, Kawakita T, Tsubota K. Effectiveness of autologous serum eye drops combined with punctal plugs for the treatment of Sjögren syndrome-related dry eye. Cornea. 2015;34(10):1214-1220.

4. Pinto-Fraga J, López-Miguel A, González-García MJ, et al. Topical Fluorometholone protects the ocular surface of dry eye patients from Desiccating stress: a randomized controlled clinical trial. Ophthalmology. 2016;123(1):141-153.

5. Park Y, Song JS, Choi C, Yoon KC, Lee HK, Kim HS. A randomized multicenter study comparing $0.1 \%, 0.15 \%$, and $0.3 \%$ Sodium Hyaluronate with $0.05 \%$ Cyclosporine in the treatment of dry eye. J Ocul Pharmacol Ther. 2016;8:122-126.

6. Lee KS, Chun SY, Lee MG, Kim S, Jang TJ, Nam KS. The prevention of TNF- $\alpha /$ IFN- $\gamma$ mixture-induced inflammation in human keratinocyte and atopic dermatitis-like skin lesions in $\mathrm{NC} / \mathrm{Nga}$ mice by mineral-balanced deep sea water. Biomed Pharmacother. 2018;97: 1331-1340.

7. Proksch E, Nissen HP, Bremgartner M, Urquhart C. Bathing in a magnesium-rich Dead Sea salt solution improves skin barrier function, enhances skin hydration, and reduces inflammation in atopic dry skin. Int J Dermatol. 2005;44(2):151-157.

8. Chen JR, Jin L, Li XY, Xy L. The effectiveness of nasal saline irrigation (seawater) in treatment of allergic rhinitis in children. Int J Pediatr Otorhinolaryngol. 2014;78(7):1115-1118.

9. Asiedu K, Kyei S, Mensah SN, Ocansey S, Abu LS, Kyere EA. Ocular surface disease index (OSDI) versus the standard patient evaluation of eye dryness (speed): a study of a nonclinical sample. Cornea. 2016; 35(2):175-180.

10. Sakane Y, Yamaguchi M, Yokoi N, et al. Development and validation of the dry Eye-Related quality-of-life score questionnaire. JAMA Ophthalmol. 2013;131(10):1331-1338.

11. Schiffman RM, Christianson MD, Jacobsen G, Hirsch JD, Reis BL. Reliability and validity of the ocular surface disease index. Arch Ophthalmol. 2000;118(5):615-621.

12. Barabino S, Labetoulle M, Rolando M, Messmer EM. Understanding symptoms and quality of life in patients with dry eye syndrome. Ocul Surf. 2016;14(3):365-376.

13. Remeseiro B, Barreira N, García-Resúa C, et al. iDEAS: a web-based system for dry eye assessment. Comput Methods Programs Biomed. 2016;130:186-197.

14. Simpson TL, Situ P, Jones LW, Fonn D. Dry eye symptoms assessed by four questionnaires. Optom Vis Sci. 2008;85(8):692-699. 
15. Tomlinson A, Khanal S, Ramaesh K, Diaper C, McFadyen A. Tear film osmolarity: determination of a referent for dry eye diagnosis. Invest Ophthalmol Vis Sci. 2006;47(10):4309-4316.

16. Abelson MB, Ousler GW 3rd, Nally LA, Welch D, Krenzer K. Alternative reference values for tear film break up time in normal and dry eye populations. Adv Exp Med Bio. 2002;506:121-125.

17. Miyake H, Kawano Y, Tanaka H, Iwata A, Imanaka T, Nakamura M. Tear volume estimation using a modified Schirmer test: a randomized, multicenter, double-blind trial comparing 3\% diquafosol ophthalmic solution and artificial tears in dry eye patients. Clin Ophthalmol. 2016; 10:879-886.

18. Akiyama-Fukuda R, Usui T, Yoshida T, Yamagami S. Evaluation of tear meniscus dynamics using anterior segment Swept-Source optical coherence tomography after topical solution instillation for dry eye. Cornea. 2016;35(5):654-658.

19. Acera A, Rocha G, Vecino E, Lema I, Durán JA. Inflammatory markers in the tears of patients with ocular surface disease. Ophthalmic Res. 2008;40(6):315-321.

20. Solomon A, Dursun D, Liu Z, Xie Y, Macri A, Pflugfelder SC. Pro- and anti-inflammatory forms of interleukin-1 in the tear fluid and conjunctiva of patients with dry-eye disease. Invest Ophthalmol Vis Sci. 2001; 42(10):2283-2292.

21. Stern ME, Gao J, Siemasko KF, Beuerman RW, Pflugfelder SC. The role of the lacrimal functional unit in the pathophysiology of dry eye. Exp Eye Res. 2004;78(3):409-416.
22. Moutsopoulos HM, Manoussakis MN. Immunopathogenesis of Sjogren's syndrome: "facts and fancy". Autoimmunity. 1989;5(1-2):17-24.

23. Chun SY, Lee KS, Nam KS. Refined deep-sea water suppresses inflammatory responses via the MAPK/AP-1 and NF- $\mathrm{KB}$.Signaling pathway in LPS-treated RAW 264. Int J Mol Sci. 2017;18:11-13.

24. Bak JP, Kim YM, Son J, Kim CJ, Kim EH. Application of concentrated deep sea water inhibits the development of atopic dermatitis-like skin lesions in NC/Nga mice. BMC Complement Altern Med. 2012;12:108.

25. Matceyevsky D, Hahoshen NY, Vexler A, Noam A, Khafif A, Ben-Yosef R. Assessing the effectiveness of dead Sea products as prophylactic agents for acute radiochemotherapy-induced skin and mucosal toxicity in patients with head and neck cancers: a phase 2 study. Isr Med Assoc J. 2007;9(6):439-442.

26. Yoshizawa Y, Tanojo H, Kim SJ, Maibach HI. Sea water or its components alter experimental irritant dermatitis in man. Skin Res Technol. 2001;7(1):36-39.

27. Cingi C, Unlu HH, Songu M, et al. Seawater gel in allergic rhinitis: entrapment effect and mucociliary clearance compared with saline. Ther Adv Respir Dis. 2010;4(1):13-18.

28. Slapak I, Skoupá J, Strnad P, Horník P. Efficacy of isotonic nasal wash (seawater) in the treatment and prevention of rhinitis in children. Arch Otolaryngol Head Neck Surg. 2008;134(1):67-74.
Clinical Ophthalmology

\section{Publish your work in this journal}

Clinical Ophthalmology is an international, peer-reviewed journal covering all subspecialties within ophthalmology. Key topics include: Optometry; Visual science; Pharmacology and drug therapy in eye diseases; Basic Sciences; Primary and Secondary eye care; Patient Safety and Quality of Care Improvements. This journal is indexed on

\footnotetext{
Submit your manuscript here: http://www.dovepress.com/clinical-ophthalmology-journal
}

\section{Dovepress}

PubMed Central and CAS, and is the official journal of The Society of Clinical Ophthalmology (SCO). The manuscript management system is completely online and includes a very quick and fair peer-review system, which is all easy to use. Visit http://www.dovepress.com/ testimonials.php to read real quotes from published authors. 